\title{
Changing fluorescein angiographic appearance of a subretinal membrane
}

\author{
GIUSEPPE GIUFFRÈ \\ From the Istituto di Clinica Oculistica dell'Università, Palermo, Italy
}

SUMMARY A patient is described in whom a post-traumatic subretinal membrane changed with time from a hypofluorescent to a hyperfluorescent appearance. It is suggested that the early hypofluorescence was due to a masking effect of the hyperplastic retinal pigment epithelium and the late hyperfluorescence to pigment defect and staining of the membrane.

Subretinal fibrosis is a clinical entity infrequently reported. It may be secondary to trauma, ${ }^{1}$ retinal detachment, ${ }^{2}$ or uveitis. ${ }^{3}$ The fluorescein angiographic features of the subretinal fibrosis are controversial, since it has been described as a lesion showing staining ${ }^{3}$ or lacking any fluorescence. ${ }^{1}$

We followed up a case of post-traumatic subretinal fibrosis for some years and record previously unreported changes in the fluorescein angiographic characteristics of the lesion.

\section{Case report}

A 47-year-old man, hit in the right eye with a thick rubber band, was observed three months after the injury. The retina showed a macular cyst and, superotemporally to the macula, a greyish, star shaped area of subretinal fibrosis (Fig. 1, left). The

Correspondence to $\mathrm{Dr}$ Giuseppe Giuffrè, Istituto di Clinica Oculistica, Via L Giuffrè 13, 90127 Palermo, Italy. fluorescein angiography in the early phase showed masking hypofluorescence of the fibrotic tissue, except for the vertices of the lesion, which displayed transmitted hyperfluorescence (Fig. 1, centre). The hyperfluorescence increased in the late venous phase, but the lesion did not show leakage (Fig. 1, right). The visual acuity was $20 / 60$.

After three years the macula developed two holes. The star-like lesion did not change its shape, though it acquired a whitish colour (Fig. 2, left). Early in fluorescein angiography the area of fibrosis showed transmitted hyperfluorescence (Fig. 2, centre), and later the hyperfluorescence increased owing to staining (Fig. 2, right). The visual acuity was 20/30, and fixation occurred along the temporal edge of the larger macular hole.

\section{Discussion}

After retinal damage the cells of the retinal pigment epithelium may proliferate in the subretinal space,

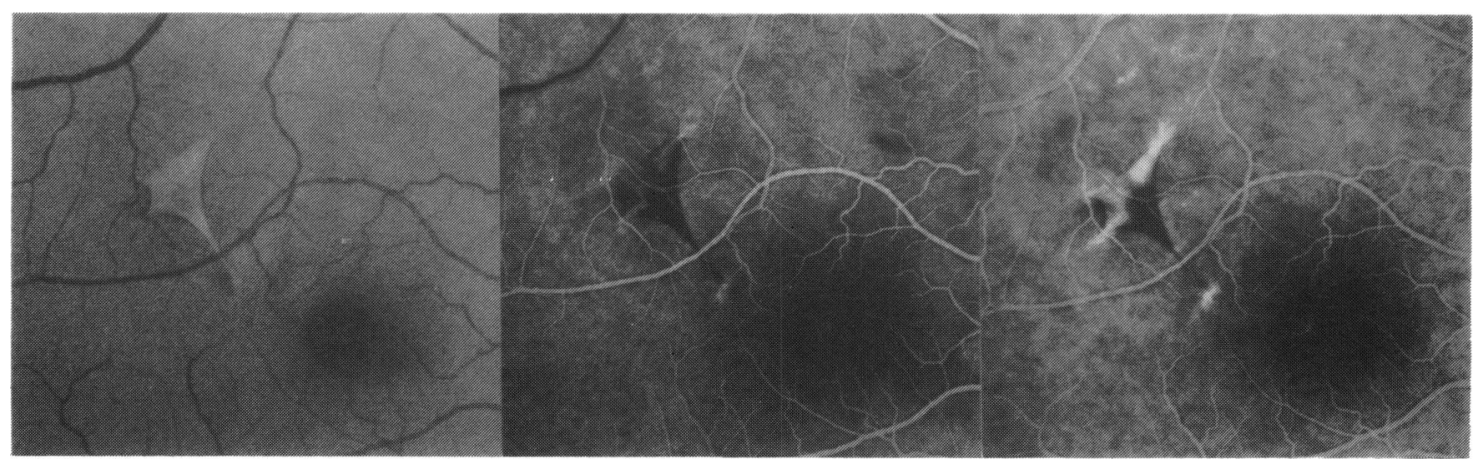

Fig. 1 The retina shows a greyish subretinal membrane and a macular cyst (left). The membrane is hypofluorescent from the early phase of fluorescein angiography (centre), but the vertices of the lesion display increasing hyperfluorescence (right). 


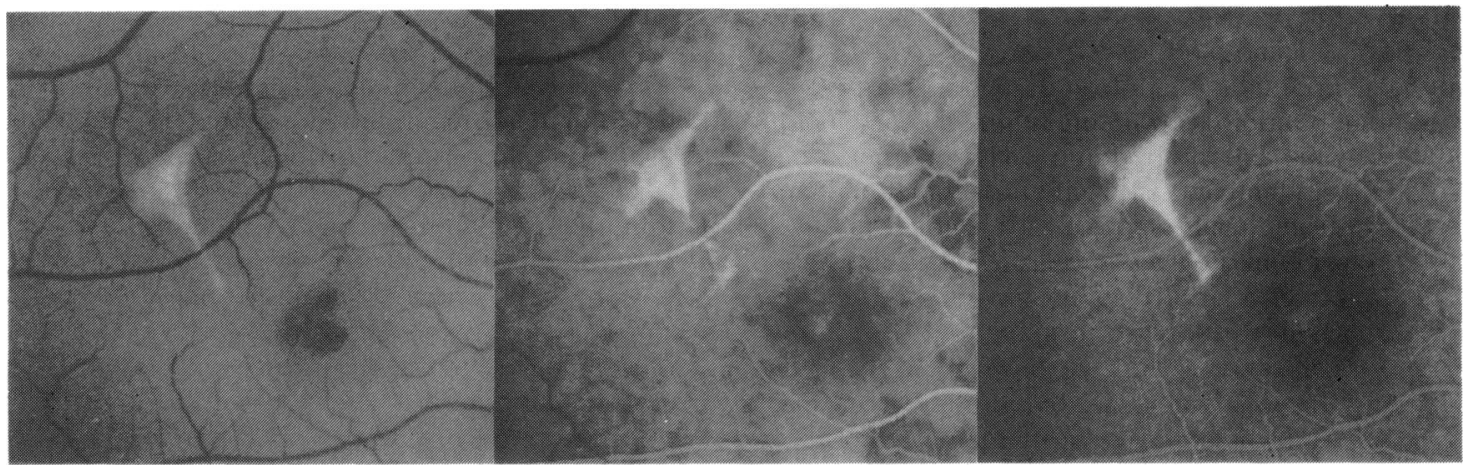

Fig. 2 After three years the subretinal membrane acquired a whitish colour and the macula developed two holes (left). The fluorescein angiography shows transmitted hyperfluorescence of the whole lesion (centre) and late staining of the membrane (right).

producing connective membranes. ${ }^{4}$ At times these pigment epithelium cells may flatten, lose their pigment granules, and degenerate, so that the main component of the membrane will be the connective tissue. $^{5}$

In the case reported here the grey subretinal membrane appeared hypofluorescent, probably because it was mainly composed of retinal pigment epithelium cells that masked the choroidal fluorescence. The vertices of the membrane, which are its oldest parts, had lost the cellular component and showed transmitted hyperfluorescence and staining. After some years, when the membrane acquired a whitish colour and was probably formed mainly of fibrous tissue, it showed hyperfluorescence from the early phases of fluorescein angiography. It can be supposed that the degeneration of the retinal pigment epithelium located under the membrane caused the early transmitted hyperfluorescence, and the staining of the connective membrane caused the late hyperfluorescence.

Thus it seems that the fluorescein angiographic appearance of the subretinal membranes can give information about the age of such membranes.

\section{References}

1 François $\mathbf{P}$. Les maculopathies traumatiques. In: François $\mathbf{P}$, Bonnet M, eds. La macula. Paris: Masson, 1976: 400-15.

2 Wallyn RH, Hilton GF. Subretinal fibrosis in retinal detachment. Arch Ophthalmol 1979; 97: 2128-9.

3 Palestine AG, Nussenblatt RB, Parver LM, Knox DL. Progressive subretinal fibrosis and uveitis. Br J Ophthalmol 1984; 68: 66773.

4 Frayer W. Reactivity of the retinal pigment epithelium: an experimental and histopathologic study. Trans Am Ophthalmol Soc 1966; 64: 586-98.

5 Tso MOM. Developmental, reactive, and neoplastic proliferation of the retinal pigment epithelium. In: Zinn KM, Marmor MK, eds. The retinal pigment epithelium. Cambridge, Mass: Harvard University Press, 1979: 267-76.

Accepted for publication 17 August 1987. 